\title{
On sums of two $k$ th powers: an asymptotic formula for the mean square of the error term
}

\author{
by
}

\section{KÜHLEITNER (Wien)}

1. Introduction. For a fixed natural number $k \geq 2$ we consider the arithmetic function $r_{k}(n)$ which counts the number of ways to write the positive integer $n$ as a sum of the $k$ th powers of two integers taken absolutely:

$$
r_{k}(n)=\#\left\{\left(u_{1}, u_{2}\right) \in \mathbb{Z}^{2}:\left|u_{1}\right|^{k}+\left|u_{2}\right|^{k}=n\right\} .
$$

To study the average order of this arithmetic function, one is interested in the Dirichlet summatory function

$$
A_{k}(t)=\sum_{1 \leq n \leq t^{k / 2}} r_{k}(n)
$$

where $t$ is a large real variable. For the special case $k=2$, Gauß proved that

$$
A_{2}(t)=\pi t+P_{2}(t),
$$

with $P_{2}(t) \ll t^{1 / 2}$. Since then the question of the exact order of $P_{2}(t)$ has been called the circle problem of Gauß. For an exposition of its history, see e.g. the textbook of Krätzel [10]. At present the sharpest upper bound is

$$
P_{2}(t)=O\left(t^{23 / 73}(\log t)^{315 / 146}\right),
$$

due to Huxley [7], [8]. In the opposite direction the best results to date are

$$
P_{2}(t)=\Omega_{-}\left((t \log t)^{1 / 4}(\log \log t)^{(\log 2) / 4} \exp (-c \sqrt{\log \log \log t})\right) \quad(c>0),
$$

and

$$
P_{2}(t)=\Omega_{+}\left(t^{1 / 4} \exp \left(c^{\prime}(\log \log t)^{1 / 4}(\log \log \log t)^{-3 / 4}\right)\right) \quad\left(c^{\prime}>0\right),
$$

due to Hafner [5], and Corrádi and Kátai [2], respectively. They refined earlier work of Hardy [6], resp. Gangadharan [3]. It is usually conjectured that

$$
\inf \left\{\theta \in \mathbb{R}: P_{2}(t) \ll_{\theta} t^{\theta}\right\}=1 / 4
$$

2000 Mathematics Subject Classification: 11P21, 11N37, 11L07. 
This hypothesis is supported by the mean square result

$$
\int_{0}^{X}\left(P_{2}(t)\right)^{2} d t=C X^{3 / 2}+O\left(X(\log X)^{2}\right), \quad C=\frac{1}{3 \pi^{2}} \sum_{n=1}^{\infty} \frac{\left(r_{2}(n)\right)^{2}}{n^{3 / 2}},
$$

which has been established (in this sharp form) by Kátai [9].

For $k \geq 3$, the asymptotic formula for $A_{k}(t)$ contains a second main term which comes from the points of the boundary curve where the curvature vanishes. It turns out that

$$
A_{k}(t)=\frac{2 \Gamma^{2}(1 / k)}{k \Gamma(2 / k)} t+B_{k} \Phi_{k}(t) t^{1 / 2-1 /(2 k)}+P_{k}(t),
$$

where

$$
\begin{aligned}
B_{k} & =2^{3-1 / k} \pi^{-1-1 / k} k^{1 / k} \Gamma\left(1+\frac{1}{k}\right), \\
\Phi_{k}(t) & =\sum_{n=1}^{\infty} n^{-1-1 / k} \sin \left(2 \pi n \sqrt{t}-\frac{\pi}{2 k}\right) .
\end{aligned}
$$

A thorough account on the history (which goes back to van der Corput [19]) and the diverse aspects of this problem can be found in the textbook of Krätzel [10]. Using Huxley's deep method in its sharpest form, Kuba [12] proved that the new error term $P_{k}(t)$ again satisfies the estimate (1.1). Quite recently Nowak [14] was able to show that this analogy partially extends to the order of the mean square, i.e.

$$
\int_{0}^{X}\left(P_{k}(t)\right)^{2} d t \ll X^{3 / 2},
$$

for a large real parameter $X$ (the $\ll$-constant possibly depending on $k$ ). Concerning lower estimates it is known that

$$
P_{k}(t)= \begin{cases}\Omega_{-}\left((t \log t)^{1 / 4}\right) & \text { for } k \geq 3, \\ \Omega_{+}\left((t \log \log t)^{1 / 4}\right) & \text { for } k=3,\end{cases}
$$

due to Nowak [15] and Nowak, Schoissengeier, Wooley and the author [13], which corresponds to the results of Hardy [6], resp. Gangadharan [3]. See also earlier works of Krätzel [11] and Schnabel [17] where somewhat weaker estimates were obtained.

The proof of the estimate (1.2) uses the fact that the generating function $\sum_{n=1}^{\infty} r_{2}(n) / n^{s}(\operatorname{Re} s>1$ ) of the number of lattice points on the circle satisfies a functional equation. For general Dirichlet series satisfying a functional equation with multiple gamma factors, Redmond [16] proved a mean-square asymptotic formula for the error term of the summatory function. In the case 
$k \geq 3$, such a handy functional equation is not available. Using a different method we improve (1.4) to

$$
\int_{0}^{X}\left(P_{k}(t)\right)^{2} d t \sim C_{k} X^{3 / 2} \quad\left(C_{k}>0\right)
$$

with an explicitly given error term.

Notation. For any fixed natural number $k$ let $q$ be defined by $1 / k+1 / q=$ 1, i.e. $q=k /(k-1)$. Further, let $|\cdot|_{q}$ denote the $q$-norm in $\mathbb{R}^{2}$, i.e.

$$
\left|\left(w_{1}, w_{2}\right)\right|_{q}=\left(\left|w_{1}\right|^{q}+\left|w_{2}\right|^{q}\right)^{1 / q} .
$$

Theorem. For any fixed integer $k \geq 3$, the error term $P_{k}(t)$ defined in (1.3) satisfies

$$
\int_{0}^{X}\left(P_{k}(t)\right)^{2} d t=C_{k} X^{3 / 2}+O\left(X^{3 / 2-\alpha_{k}+\varepsilon}\right)
$$

for any $\varepsilon>0$, where

$$
C_{k}=\frac{16}{3 \pi^{2}(k-1)} \sum_{\substack{a, b, c, d \in \mathbb{N} \\|(a, b)|_{q}=|(c, d)|_{q}}}(a b c d)^{-1+q / 2}|(a, b)|_{q}^{-2 q+1}
$$

and

$$
\alpha_{k}=\frac{1}{12\left(q+1 / 6+k(k-1)^{4}\right)} .
$$

REMARKs. 1. The convergence of the above series will be a by-result of our proof: see (3.15) ff.

2 . The constant $\alpha_{k}$ can certainly be improved by a more elaborate analysis. The author did not invest much effort to obtain the optimal $\alpha_{k}$ in reach of the present method.

3. It is natural to compare our constant $C_{k}$ with the constant $C$ of (1.2) which may be written in the form

$$
C=\frac{1}{3 \pi^{2}} \sum_{\substack{a, b, c, d \in \mathbb{Z} \\ 0<|(a, b)|_{2}=|(c, d)|_{2}}}|(a, b)|_{2}^{-3} .
$$

We notice that this latter sum ranges also over the pairs $(a, b),(c, d)$ with one vanishing component, in contrast to the series for $C_{k}, k \geq 3$. The reason for this is that the Lamé's curve has curvature 0 at its points of intersection with the coordinate axes: These give rise to the second main term in (1.3). 


\section{Some lemmas}

Lemma 1 (see Vaaler [18]). For arbitrary $w \in \mathbb{R}$ and $H \in \mathbb{N}$, let

$$
\psi(w)=w-[w]-1 / 2, \quad \psi_{H}^{*}(w)=-\frac{1}{\pi} \sum_{h=1}^{H} \frac{\sin (2 \pi h w)}{h} \tau\left(\frac{h}{H+1}\right),
$$

where

$$
\tau(x)=\pi x(1-x) \cot (\pi x)+x \quad \text { for } 0<x<1
$$

Then

$$
\left|\psi(w)-\psi_{H}^{*}(w)\right| \leq \frac{1}{H+1} \sum_{h=1}^{H}\left(1-\frac{h}{H+1}\right) \cos (2 \pi h w)+\frac{1}{2 H+2} .
$$

LEMMA 2. Let $f(w)$ be a real-valued function with continuous derivatives up to the fourth order on the interval $[A, B]$. Let $L$ and $U$ be real parameters not less than 1 such that $B-A \asymp L$,

$$
f^{(j)}(w) \ll U L^{1-j} \quad \text { for } w \in[A, B], j=1,2,3,4,
$$

and, for some $C>0$,

$$
f^{\prime \prime}(w) \geq C U L^{-1} \quad \text { for } w \in[A, B] .
$$

Suppose that $f^{\prime}(A)$ and $f^{\prime}(B)$ are integers, and denote by $\phi$ the inverse function of $f^{\prime}$. Then

$$
\begin{aligned}
\sum_{A \leq n \leq B} e(f(n))= & e\left(\frac{1}{8}\right) \sum_{f^{\prime}(A) \leq m \leq f^{\prime}(B)}^{\prime \prime} \frac{e(f(\phi(m))-m \phi(m))}{\sqrt{f^{\prime \prime}(\phi(m))}} \\
& +O(\log (1+U)),
\end{aligned}
$$

where $e(u)=e^{2 \pi i u}$ for real $u$, and $\sum^{\prime \prime}$ indicates that the terms corresponding to $m=f^{\prime}(A)$, resp. $m=f^{\prime}(B)$ are weighted with the factor $1 / 2$. The implied $O$-constant depends at most on $C$ and on the constants implied in the order symbols in the suppositions.

To prove Lemma 2, let us first state the following result.

LEMMA 3. Let $F(w)$ be a real-valued function with continuous derivatives up to the fourth order on the interval $[A, B]$. Let $L$ and $U$ be real parameters not less than 1 such that $B-A \asymp L$,

$$
F^{(j)}(w) \ll U L^{1-j} \quad \text { for } w \in[A, B], j=1,2,3,4,
$$

and, for some $C>0$,

$$
F^{\prime \prime}(w) \geq C U L^{-1} \quad \text { for } w \in[A, B] .
$$


Suppose that there exists a value $c \in[A, B]$ for which $F^{\prime}(c)=0$. Then

$$
\begin{aligned}
& \int_{A}^{B} e(F(w)) d w \\
& = \begin{cases}\frac{1}{2}\left(F^{\prime \prime}(A)\right)^{-1 / 2} e\left(\frac{1}{8}+F(A)\right)+O\left(\frac{1}{\left|F^{\prime}(B)\right|}\right)+O\left(\frac{1}{U}\right) & \text { if } c=A, \\
\frac{1}{2}\left(F^{\prime \prime}(B)\right)^{-1 / 2} e\left(\frac{1}{8}+F(B)\right)+O\left(\frac{1}{\left|F^{\prime}(A)\right|}\right)+O\left(\frac{1}{U}\right) & \text { if } c=B, \\
\left(F^{\prime \prime}(c)\right)^{-1 / 2} e\left(\frac{1}{8}+F(c)\right)+O\left(\frac{1}{\left|F^{\prime}(A)\right|}+\frac{1}{\left|F^{\prime}(B)\right|}\right)+O\left(\frac{1}{U}\right) & \text { else. }\end{cases}
\end{aligned}
$$

Proof. For $A<c<B$, this is explicitly contained in Lemma 3.4 of Graham and Kolesnik [4]. The case $c=B$ can be reduced to $c=A$ by the substitution $w \mapsto A+B-w$. Finally, to deal with the case $c=A$, it suffices to have a close look at the proof of Lemma 3.4 in [4]: Here $F$ is approximated by its quadratic Taylor expansion $q(w)$ at the stationary point $c$. If $c=A$, the integral $\int_{A}^{B} e(q(w)) d w$ can be evaluated by an obvious variant of Lemma 3.3 in [4], viz.

$$
\int_{0}^{X} e\left(H w^{2}\right) d w=\frac{e(1 / 8)}{2 \sqrt{2 H}}+O\left(\frac{1}{H X}\right) .
$$

The ingenious estimation of the remainder integral then works exactly as in [4].

Proof of Lemma 2. Again by [4], Lemma 3.5 (after taking conjugates),

$$
\sum_{A \leq n \leq B} e(f(n))=\sum_{m=f^{\prime}(A)-1}^{f^{\prime}(B)+1} \int_{A}^{B} e(f(w)-m w) d w+O(\log (1+U)) .
$$

To each of the integrals on the right-hand side we apply Lemma 3. (The first and last one are estimated as $O(1)$ by the first derivative test.) The main term produces no difficulties, and the error terms are readily

$$
\begin{aligned}
& \ll \frac{1}{f^{\prime}(B)-f^{\prime}(A)}+\sum_{m=f^{\prime}(A)+1}^{f^{\prime}(B)-1}\left(\frac{1}{m-f^{\prime}(A)}+\frac{1}{f^{\prime}(B)-m}\right) \\
& \quad+\frac{1}{U}\left(f^{\prime}(B)-f^{\prime}(A)+1\right) \\
& \ll \log (1+U) .
\end{aligned}
$$

LEMma 4. Let $s, t, u, v$ be natural numbers with

$$
|(s, t)|_{q}-|(u, v)|_{q} \neq 0 .
$$


Then

$$
\|\left.(s, t)\right|_{q}-|(u, v)|_{q} \mid \gg M^{-\left(q-1+k(k-1)^{4}\right)},
$$

where $M=\max (s, t, u, v)$ and the implied $\gg$ constant depends at most on $k$.

Proof. By the mean value theorem we have

$$
|(s, t)|_{q}^{q}-\left.|(u, v)|_{q}^{q} \ll M^{q-1}||(s, t)\right|_{q}-|(u, v)|_{q} \mid .
$$

The left-hand side of (2.1) can be written as

$$
L:=a^{1 / r}+b^{1 / r}-c^{1 / r}-d^{1 / r} \neq 0,
$$

with natural numbers $a=s^{k}, b=t^{k}, c=u^{k}, d=v^{k}$, and $r:=k-1$. Consider the field extension

$$
\mathbb{F}=\mathbb{Q}\left(a^{1 / r}, b^{1 / r}, c^{1 / r}, d^{1 / r}, e^{2 \pi i / r}\right) .
$$

The corresponding Galois group $G=\operatorname{Gal}(\mathbb{F} / \mathbb{Q})$ contains at most $r^{5}$ elements $\chi$. It is clear that

$$
\left|\prod_{\chi \in G} \chi(L)\right| \geq 1
$$

since the left-hand side is the modulus of the norm of a nonzero algebraic integer. Furthermore, for every $\chi \in G$,

$$
|\chi(L)| \leq\left|\chi\left(a^{1 / r}\right)\right|+\left|\chi\left(b^{1 / r}\right)\right|+\left|\chi\left(c^{1 / r}\right)\right|+\left|\chi\left(d^{1 / r}\right)\right| \leq 4 M^{k / r} .
$$

Consequently,

$$
|L| \geq \prod_{\substack{\chi \in G \\ \chi \neq \mathrm{id}}}|\chi(L)|^{-1} \gg M^{-r^{4}},
$$

which establishes Lemma 4.

3. Proof of the Theorem. We use $\varepsilon$ to denote an arbitrary small positive quantity which need not be the same at each occurrence. The constants implied in the symbols $\ll$ and $O$ may depend on $\varepsilon$ and $k$. We start from formulae (3.57), (3.58) of Krätzel [10], p. 148, and the asymptotic expansion below:

$$
P_{k}(t)=-8 \sum_{\alpha \sqrt{t} \leq n \leq \sqrt{t}} \psi\left(\left(t^{k / 2}-n^{k}\right)^{1 / k}\right)+O(1),
$$

with $\psi(w)=w-[w]-1 / 2$ throughout, and $\alpha:=2^{-1 / k}$.

In what follows, let $T$ be sufficiently large and $t \in[T, 2 T]$. We split up the domain of summation into subintervals $\mathcal{N}_{j}(t)=\left[N_{j}, N_{j+1}\right]$, where

$$
N_{j}=\frac{\sqrt{t}}{\left(1+2^{-j q}\right)^{1 / k}}, \quad j=0,1, \ldots, J
$$


with $J$ minimal such that $\sqrt{t}-N_{J}<1$ for $T \leq t \leq 2 T$. Thus

$$
P_{k}(t)=-8 \sum_{j=0}^{J} \sum_{n \in \mathcal{N}_{j}(t)} \psi\left(\left(t^{k / 2}-n^{k}\right)^{1 / k}\right)+O(\log T) .
$$

Further let

$$
P_{k}^{*}(t)=-8 \sum_{j=0}^{J} \sum_{n \in \mathcal{N}_{j}(t)} \psi_{H}^{*}\left(\left(t^{k / 2}-n^{k}\right)^{1 / k}\right),
$$

with $\psi_{H}^{*}(w)$ defined as in Lemma 1.

We will prove the following Proposition. By applying Cauchy's inequality and summing over $T=X / 2, X / 4, \ldots$, our Theorem readily follows.

Proposition. For sufficiently large $T$ and $H=\left[T^{1 / 4+\alpha_{k}}\right]$, with $\alpha_{k}$ defined as in (1.5), we have

$$
\begin{aligned}
& \int_{T}^{2 T}\left|P_{k}(t)-P_{k}^{*}(t)\right|^{2} d t \ll T^{3 / 2-2 \alpha_{k}}, \\
& \int_{T}^{2 T}\left|P_{k}^{*}(t)\right|^{2} d t=\mathcal{H}(T)+O\left(T^{3 / 2-\alpha_{k}+\varepsilon}\right),
\end{aligned}
$$

with

$$
\mathcal{H}(T)=\frac{8}{\pi^{2}(k-1)} \sum_{\substack{a, b, c, d \in \mathbb{N} \\|(a, b)|_{q}=|(c, d)|_{q}}}(a b c d)^{-1+q / 2}|(a, b)|_{q}^{-2 q+1} \int_{T}^{2 T} t^{1 / 2} d t .
$$

Proof. (i) By (3.1), (3.2) and Lemma 1, the left-hand side of (i) is

$$
\begin{aligned}
& \ll \int_{T}^{2 T}\left(\frac{1}{H^{2}} \sum_{1 \leq h \leq H} \sum_{j=0}^{J} \sum_{n \in \mathcal{N}_{j}(t)} \cos \left(-2 \pi h\left(t^{k / 2}-n^{k}\right)^{1 / k}\right)\right)^{2} d t \\
& \quad+O\left(T^{3 / 2-2 \alpha_{k}}\right) .
\end{aligned}
$$

By Cauchy's inequality, it thus suffices to show that there exists a constant $c_{0}>1$ such that, for $T$ sufficiently large and $0 \leq j \leq J$,

$$
\begin{aligned}
I_{j}(T) & :=\int_{T}^{2 T}\left(\frac{1}{H^{2}} \sum_{1 \leq h \leq H} \sum_{n \in \mathcal{N}_{j}(t)} \cos \left(-2 \pi h\left(t^{k / 2}-n^{k}\right)^{1 / k}\right)\right)^{2} d t \\
& \ll c_{0}^{-j} T^{3 / 2-2 \alpha_{k}} .
\end{aligned}
$$

We transform each of the inner trigonometric sums over $n$ by Lemma 2, with $[A, B]=\left[N_{j}, N_{j+1}\right]$, and

$$
f(w)=-h\left(t^{k / 2}-w^{k}\right)^{1 / k} .
$$


We note that $f^{\prime}\left(N_{j}\right)$ is independent of $t$, more precisely

$$
f^{\prime}\left(N_{j}\right)=h 2^{j} \text { and } \quad N_{j+1}-N_{j} \asymp \sqrt{T} / 2^{j q} .
$$

Calculating derivatives, we get

$$
\begin{aligned}
f^{(1)}(w)= & h w^{k-1}\left(t^{k / 2}-w^{k}\right)^{-1+1 / k} \\
f^{(2)}(w)= & h(k-1) t^{k / 2} w^{k-2}\left(t^{k / 2}-w^{k}\right)^{-2+1 / k} \\
\asymp & h T^{1 / 2-1 /(2 k)}(\sqrt{t}-w)^{-2+1 / k} \\
\asymp & h T^{1 / 2-1 /(2 k)}\left(\sqrt{T} / 2^{j q}\right)^{-2+1 / k} \\
\asymp & h T^{-1 / 2} 2^{j q(2-1 / k)} \\
f^{(3)}(w)= & h(k-1) t^{k / 2} w^{k-3}\left(t^{k / 2}-w^{k}\right)^{-3+1 / k}\left((k-2) t^{k / 2}+(k+1) w^{k}\right) \\
\asymp & h T^{1 / 2-1 /(2 k)}(\sqrt{t}-w)^{-3+1 / k} \\
\asymp & h T^{-1} 2^{j q(3-1 / k)}, \\
f^{(4)}(w)= & h(k-1) t^{k / 2} w^{k-4}\left(t^{k / 2}-w^{k}\right)^{-4+1 / k} \\
& \times\left((k-2) t^{k / 2}\left((k-3) t^{k / 2}+2(k+1) w^{k}\right)\right. \\
& \left.+(1+k) w^{k}\left((2 k-3) t^{k / 2}+(k+2) w^{k}\right)\right) \\
\asymp & h T^{1 / 2-1 /(2 k)}(\sqrt{t}-w)^{-4+1 / k} \\
\asymp & h T^{-3 / 2} 2^{j q(-4+1 / k)} .
\end{aligned}
$$

One easily verifies that the conditions of Lemma 2 are satisfied, with $L=$ $\sqrt{T} 2^{-j q}$ and $U=h 2^{j}$. We note that in view of $(3.4) f^{\prime}\left(N_{j}\right)$ and $f^{\prime}\left(N_{j+1}\right)$ are integers. We may thus apply the lemma to conclude by a straightforward calculation that, for $T \leq t \leq 2 T$,

$$
\begin{aligned}
& \sum_{n \in \mathcal{N}_{j}(t)} e\left(-h\left(t^{k / 2}-n^{k}\right)^{1 / k}\right) \\
& =\frac{e(1 / 8)}{\sqrt{k-1}} h t^{1 / 4} \sum_{m \in \mathcal{M}_{j}(h)}^{\prime \prime}(h m)^{-1+q / 2}|(h, m)|_{q}^{-q+1 / 2} e\left(-\sqrt{t}|(h, m)|_{q}\right) \\
& \quad+O(j+\log h),
\end{aligned}
$$

where

$$
\mathcal{M}_{j}(h)=\left[f^{\prime}\left(N_{j}\right), f^{\prime}\left(N_{j+1}\right)\right]
$$

Therefore, using the real part of (3.5), we obtain

$$
I_{j}(T) \ll I_{j}^{*}(T)+T(\log T)^{4}
$$

with

$$
I_{j}^{*}(T):=\int_{T}^{2 T} \frac{t^{1 / 2}}{H^{2}}\left|\sum_{1 \leq h \leq H} S_{h}(t)\right|^{2} d t
$$


and

$$
S_{h}(t):=\sum_{m \in \mathcal{M}_{j}(h)}^{\prime \prime} h(h m)^{-1+q / 2}|(h, m)|_{q}^{-q+1 / 2} e\left(\sqrt{t}|(h, m)|_{q}\right) .
$$

To estimate the integral in (3.7) we follow the proof of Nowak [14]. We split up the domain of summation over $h$ into dyadic subintervals: Let

$$
\left.\left.\mathcal{H}_{i}=\right] H_{i+1}, H_{i}\right], \quad H_{i}=H / 2^{i}, \quad i=0,1, \ldots, I,
$$

where $I$ is the largest integer for which $2^{I}<H$. By Cauchy's inequality,

$$
\left|\sum_{h=1}^{H} S_{h}(t)\right|^{2} \ll \sum_{i=0}^{I} H_{i}^{\varepsilon}\left|\sum_{h \in \mathcal{H}_{i}} S_{h}(t)\right|^{2}
$$

with some fixed $\varepsilon>0$ sufficiently small.

In what follows, we write $\mathbf{u}=\left(u_{1}, u_{2}\right), \mathbf{v}=\left(v_{1}, v_{2}\right)$ for elements of $\mathbb{Z}^{2}$, and put

$$
\mathcal{U}_{i, j}:=\left\{(h, m): h \in \mathcal{H}_{i}, m \in \mathcal{M}_{j}(h)\right\} .
$$

By squaring and integrating term by term we get

$$
\begin{aligned}
\int_{T}^{2 T} \frac{t^{1 / 2}}{H^{2}}\left|\sum_{h \in \mathcal{H}_{i}} S_{h}(t)\right|^{2} d t & \\
& \ll \frac{T^{1 / 2}}{H^{2}} \sum_{\mathbf{u}, \mathbf{v} \in \mathcal{U}_{i, j}} \frac{u_{1} v_{1}\left(|\mathbf{u}|_{q}|\mathbf{v}|_{q}\right)^{-q+1 / 2}}{\left(u_{1} u_{2} v_{1} v_{2}\right)^{1-q / 2}}\left|\int_{T}^{2 T} e\left(\sqrt{t}\left(|\mathbf{u}|_{q}-|\mathbf{v}|_{q}\right)\right) d t\right| .
\end{aligned}
$$

Recalling $f^{\prime}\left(N_{j}\right)=h 2^{j}$ we conclude that $\mathbf{u}=\left(u_{1}, u_{2}\right) \in \mathcal{U}_{i, j}$ implies that

$$
u_{1} \asymp H_{i}, \quad|\mathbf{u}|_{q} \asymp u_{2} \asymp H_{i} 2^{j} .
$$

Consequently,

$$
\begin{aligned}
\int_{T}^{2 T} \frac{t^{1 / 2}}{H^{2}} \mid & \left.\sum_{h \in \mathcal{H}_{i}} S_{h}(t)\right|^{2} d t \\
& \ll \frac{T^{1 / 2}}{H^{2}} H_{i}^{-1} 2^{-j(1+q)} \sum_{\mathbf{u} \in \mathcal{U}_{i, j}}\left(\sum_{\mathbf{v}:|\mathbf{v}|_{q} \leq|\mathbf{u}|_{q}} \min \left(T, \frac{\sqrt{T}}{|\mathbf{u}|_{q}-|\mathbf{v}|_{q}}\right)\right)
\end{aligned}
$$

For the inner sum over $\mathbf{v}$ we have the estimate

$$
\sum_{\mathbf{v}:|\mathbf{v}|_{q} \leq|\mathbf{u}|_{q}} \min \left(T, \frac{\sqrt{T}}{|\mathbf{u}|_{q}-|\mathbf{v}|_{q}}\right) \ll|\mathbf{u}|_{q} \sqrt{T} \log T+T|\mathbf{u}|_{q}^{2 / 3}
$$

(see Nowak [14], formula (2.14) and below). Inserting this in (3.9), and recalling (3.8) we obtain 


$$
\begin{aligned}
\int_{T}^{2 T} \frac{t^{1 / 2}}{H^{2}} \mid \sum_{h \in \mathcal{H}_{i}} & \left.S_{h}(t)\right|^{2} d t \\
& \ll \frac{T^{1 / 2}}{H^{2}} H_{i}^{-1} 2^{-j(q+1)} H_{i}^{2} 2^{j}\left(\sqrt{T} \log T H_{i} 2^{j}+T\left(H_{i} 2^{j}\right)^{2 / 3}\right) \\
& \ll T \log T 4^{-i} 2^{-j(q-1)}+T^{17 / 12-\alpha_{k} / 3} 2^{-5 /(3 i)} 2^{-j(q-2 / 3)} .
\end{aligned}
$$

Therefore,

$$
I_{j}^{*}(T) \ll T^{5 / 4} 2^{-j(q-1)}+T^{3 / 2-2 \alpha_{k}} 2^{-j(q-2 / 3)} .
$$

In view of (3.6) this proves (3.3) and therefore part (i) of the Proposition.

(ii) We insert (3.2) and the definition of $\psi_{H}^{*}(\cdot)$ into the left-hand side of (ii), transform the inner sums over $n$ by Lemma 2, and take the imaginary part of (3.5) to obtain

$$
\int_{T}^{2 T}\left(P_{k}^{*}(t)\right)^{2} d t=\frac{64}{\pi^{2}(k-1)} \int_{T}^{2 T} t^{1 / 2}\left(S_{h}^{*}(t)\right)^{2} d t+O\left(T^{5 / 4}(\log T)^{3}\right),
$$

where

$$
\begin{aligned}
S_{h}^{*}(t):= & \sum_{(h, m) \in \mathcal{D}(T)}^{\prime} \tau\left(\frac{h}{H+1}\right)(h m)^{-1+q / 2}|(h, m)|_{q}^{-q+1 / 2} \\
& \times \cos \left(2 \pi \sqrt{t}|(h, m)|_{q}+\pi / 4\right),
\end{aligned}
$$

and $H=\left[T^{1 / 4+\alpha_{k}}\right]$, anticipating that the first term of (3.10) is bounded by $O\left(T^{3 / 2}\right)$. The domain of summation is given by

$$
\mathcal{D}(T)=\left\{(h, m) \in \mathbb{N}^{2}: 1 \leq h \leq T^{1 / 4+\alpha_{k}}, h \leq m \leq f^{\prime}\left(N_{J}\right)\right\},
$$

and $\sum^{\prime}$ indicates that the terms corresponding to $h=m$ are weighted with the factor $1 / 2$. For a large real parameter $M$, we define the set

$$
\mathcal{B}(M):=\left\{(h, m) \in \mathbb{N}^{2}: h \leq m,|(h, m)|_{q} \leq M\right\},
$$

such that $\mathcal{B}(M) \subset \mathcal{D}(T)$. We write the sum $S_{h}^{*}(t)$ as

$$
\begin{gathered}
\left\{\sum_{(h, m) \in \mathcal{B}(M)}^{\prime}+\sum_{\substack{(h, m) \in \mathcal{D}(T) \\
|(h, m)|_{q}>M}}^{\prime}\right\} \tau\left(\frac{h}{H+1}\right)(h m)^{-1+q / 2}|(h, m)|_{q}^{-q+1 / 2} \\
\quad \times \cos \left(2 \pi \sqrt{t}|(h, m)|_{q}+\pi / 4\right) \\
=: \Sigma_{1}(t)+\Sigma_{2}(t) .
\end{gathered}
$$

In what follows we choose

$$
M=T^{6 \alpha_{k}} .
$$


Let us first consider

$$
\int_{T}^{2 T} t^{1 / 2}\left(\Sigma_{2}(t)\right)^{2} d t
$$

Repeating the proof of (3.7) above, with $S_{h}(T)$ replaced by $\Sigma_{2}(t)$, we conclude with the notation there that

$$
\begin{aligned}
\int_{T}^{2 T} t^{1 / 2}\left(\sum_{h \in \mathcal{H}_{i}} \Sigma_{2}(t)\right)^{2} d t & \ll 2^{-j(q-1)} T \log T+2^{-j(q-2 / 3)} H_{i}^{-1 / 3} T^{3 / 2} \\
& \ll 2^{-j(q-1)} T \log T+M^{-1 / 3+\varepsilon} H_{i}^{-\varepsilon} 2^{-j /(k-1)} T^{3 / 2},
\end{aligned}
$$

with a fixed positive $\varepsilon>0$. Therefore, (3.12) is $\ll T^{3 / 2-2 \alpha_{k}+\varepsilon}$.

By (3.10) and the Cauchy-Schwarz inequality, this implies that, again anticipating that the main term on the right-hand side of (3.10) is $O\left(T^{3 / 2}\right)$, we have

$$
\int_{T}^{2 T}\left(P_{k}^{*}(t)\right)^{2} d t=\frac{64}{\pi^{2}(k-1)} \int_{T}^{2 T} t^{1 / 2}\left(\Sigma_{1}(t)\right)^{2} d t+O\left(T^{3 / 2-\alpha_{k}+\varepsilon}\right) .
$$

The next step is to get rid of Vaaler's smoothing factors $\tau(\cdot)$, i.e. to approximate $\Sigma_{1}(t)$ by

$$
\Sigma(t):=\sum_{(h, m) \in \mathcal{B}(M)}^{\prime}(h m)^{-1+q / 2}|(h, m)|_{q}^{-q+1 / 2} \cos \left(2 \pi \sqrt{t}|(h, m)|_{q}+\pi / 4\right) .
$$

In view of the Taylor expansion $\tau(x)=1+O\left(x^{2}\right)$ for $x \rightarrow 0$, and the estimate

$$
\begin{aligned}
\sum_{(h, m) \in \mathcal{B}(M)}(h m)^{-1+q / 2}|(h, m)|_{q}^{-q+1 / 2} & \\
& \ll \sum_{m \leq M} \sum_{h \leq m} m^{-1 / 2-q / 2} h^{-1+q / 2} \ll M^{1 / 2}
\end{aligned}
$$

it follows that

$$
\Sigma(t)-\Sigma_{1}(t) \ll T^{-1 / 2+13 \alpha_{k}} .
$$

We therefore conclude that

$$
\int_{T}^{2 T}\left(P_{k}^{*}(t)\right)^{2} d t=\frac{64}{\pi^{2}(k-1)} \int_{T}^{2 T} t^{1 / 2}(\Sigma(t))^{2} d t+O\left(T^{3 / 2-\alpha_{k}+\varepsilon}\right) .
$$

Write $\mathbf{u}=\left(u_{1}, u_{2}\right), \mathbf{v}=\left(v_{1}, v_{2}\right)$ for elements of $\mathbb{N}^{2} \cap\left\{\left(w_{1}, w_{2}\right): w_{1} \leq w_{2}\right\}$. Squaring out $(\Sigma(t))^{2}$ and using the elementary formula

$$
\cos A \cos B=\frac{1}{2}(\cos (A-B)+\cos (A+B)),
$$

we can write

$$
(\Sigma(t))^{2}:=S_{0}-S_{1}(T)+S_{2}(t, T)+S_{3}(t, T),
$$


where

$$
\begin{aligned}
S_{0}:= & \frac{1}{2} \sum_{\substack{|\mathbf{u}|_{q}=|\mathbf{v}|_{q} \\
u_{1} \leq u_{2}, v_{1} \leq v_{2}}}^{\prime}\left(u_{1} u_{2} v_{1} v_{2}\right)^{-1+q / 2}\left(|\mathbf{u}|_{q}|\mathbf{v}|_{q}\right)^{-q+1 / 2}, \\
S_{1}(T):= & \frac{1}{2} \sum_{\substack{\mathbf{u}, \mathbf{v} \notin \mathcal{B}(M) \\
|\mathbf{u}|_{q}=|\mathbf{v}|_{q}}}^{\prime}\left(u_{1} u_{2} v_{1} v_{2}\right)^{-1+q / 2}\left(|\mathbf{u}|_{q}|\mathbf{v}|_{q}\right)^{-q+1 / 2}, \\
S_{2}(t, T):= & \frac{1}{2} \sum_{\substack{\mathbf{u}, \mathbf{v} \in \mathcal{B}(M) \\
|\mathbf{u}|_{q} \neq|\mathbf{v}|_{q}}}^{\prime}\left(u_{1} u_{2} v_{1} v_{2}\right)^{-1+q / 2}\left(|\mathbf{u}|_{q}|\mathbf{v}|_{q}\right)^{-q+1 / 2} \\
& \times \cos \left(2 \pi \sqrt{t}\left(|\mathbf{u}|_{q}-|\mathbf{v}|_{q}\right)\right), \\
S_{3}(t, T):= & \frac{1}{2} \sum_{\substack{\mathbf{u}, \mathbf{v} \in \mathcal{B}(M)\\
}} \times \cos \left(2 \pi \sqrt{t}\left(|\mathbf{u}|_{q}+|\mathbf{v}|_{q}\right)+\pi / 2\right) .
\end{aligned}
$$

We will show that the main term on the left-hand side of (3.14) comes from $S_{0}$. Indeed, the contribution of $S_{2}(t, T)$ is

$$
\ll \sum_{\substack{|\mathbf{u}|_{q},|\mathbf{v}|_{q} \leq M \\|\mathbf{u}|_{q} \neq\left.\mathbf{v}\right|_{q}}}\left(u_{1} u_{2} v_{1} v_{2}\right)^{-1+q / 2}\left(|\mathbf{u}|_{q}|\mathbf{v}|_{q}\right)^{-q+1 / 2} \frac{T}{\left.|| \mathbf{u}\right|_{q}-|\mathbf{v}|_{q} \mid} \ll T^{3 / 2-\alpha_{k}}
$$

by (3.13), since

$$
\int_{T}^{2 T} t^{1 / 2} \cos \left(2 \pi \sqrt{t}\left(|\mathbf{u}|_{q}-|\mathbf{v}|_{q}\right)\right) d t \ll \frac{T}{\left.|| \mathbf{u}\right|_{q}-|\mathbf{v}|_{q} \mid} \ll T M^{q-1+k(k-1)^{4}}
$$

by Lemma 4 . The contribution of $S_{3}(t, T)$ is clearly not more than this. Finally consider the contribution of $S_{1}(T)$. For positive integers $u_{1}, u_{2}, v_{1}$, $v_{2}$ with $u_{1} \leq u_{2}, v_{1} \leq v_{2}$, the condition $|\mathbf{u}|_{q}=|\mathbf{v}|_{q}$ is satisfied if and only if either $\left(u_{1}, u_{2}\right)=\left(v_{1}, v_{2}\right)$ or $u_{1}, u_{2}, v_{1}, v_{2}$ all have the same maximal $(k-1)$-free divisor $r$, say, i.e.,

$$
u_{1}=a^{k-1} r, \quad u_{2}=b^{k-1} r, \quad v_{1}=c^{k-1} r, \quad v_{2}=d^{k-1} r,
$$

with $a, b, c, d \in \mathbb{N}$ satisfying $a^{k}+b^{k}=c^{k}+d^{k}$. This follows from the fact that the $(k-1)$ th roots of distinct $(k-1)$-free positive integers are linearly independent over $\mathbb{Q}$ (see Besicovitch [1]). Therefore,

$$
S_{1}(T) \ll \sum_{\substack{u_{1}=1 \\ u_{2} \gg M}}^{\infty}\left(u_{1} u_{2}\right)^{-2+q}\left(u_{1} u_{2}\right)^{-q+1 / 2}+\mathcal{R}(T),
$$


with

$$
\begin{aligned}
\mathcal{R}(T)= & \sum_{\substack{a \leq b, c \leq d \\
b^{k-1} r, d^{k-1} r \gg M}}(a b c d)^{(k-1)(-1+q / 2)} r^{-3} \\
& \times\left(\left|\left(a^{k-1}, b^{k-1}\right)\right|_{q}\left|\left(c^{k-1}, d^{k-1}\right)\right|_{q}\right)^{-q+1 / 2},
\end{aligned}
$$

since $\left|\left(u_{1}, u_{2}\right)\right|_{q}=r\left|\left(a^{k-1}, b^{k-1}\right)\right|_{q}$. The first term in (3.15) is $\ll M^{-1 / 2}$. We estimate $\mathcal{R}(T)$ in the cases $k=3,4$, resp. $k \geq 5$ in two different ways. In the first case we use

$$
\frac{1}{\left|\left(x^{k-1}, y^{k-1}\right)\right|_{q}^{q-1 / 2}} \ll(x y)^{-\frac{1}{2}(k-1)(q-1 / 2)},
$$

to get

$$
\begin{aligned}
\mathcal{R}(T) & \ll \sum_{b^{k-1} r, d^{k-1} r \gg M} \sum_{a, c=1}^{\infty}(a b c d)^{(k-1)(-1+q / 2)} r^{-3}(a b c d)^{-\frac{1}{2}(k-1)(q-1 / 2)} \\
& \ll\left(\sum_{b^{k-1} r \gg M} r^{-3 / 2} b^{-\frac{3}{4}(k-1)}\right)^{2} \ll\left(\sum_{r=1}^{\infty} r^{-3 / 2} \sum_{b^{k-1} \gg M / r} b^{-\frac{3}{4}(k-1)}\right)^{2} \\
& \ll M^{-1 / 2} .
\end{aligned}
$$

In the case $k \geq 5$ we use the fact that

$$
\sum_{a, c=1}^{\infty}(a c)^{(k-1)(-1+q / 2)} \ll 1
$$

to get

$$
\begin{aligned}
\mathcal{R}(T) & \ll \sum_{b^{k-1} r, d^{k-1} r \gg M}(b d)^{(k-1)(-1+q / 2-q+1 / 2)} r^{-3} \\
& \ll\left(\sum_{b=1}^{\infty} b^{-k+1 / 2} \sum_{r \gg M / b^{k-1}} r^{-3 / 2}\right)^{2} \ll M^{-1} .
\end{aligned}
$$

By (3.11), we therefore conclude that

$$
S_{1}(T) \int_{T}^{2 T} t^{1 / 2} d t \ll T^{3 / 2-\alpha_{k}},
$$

which completes the proof of (ii).

Acknowledgements. The author wishes to express his sincere gratitude to Prof. Dr. W. G. Nowak for many valuable remarks and helpful comments on various parts of this work, especially for making available the ingenious version of van der Corput's transform (Lemma 2), which is taken from a forthcoming joint paper of Huxley, Krätzel and Nowak. 


\section{References}

[1] A. S. Besicovitch, On the linear independence of fractional powers of integers, J. London Math. Soc. 15 (1940), 3-6.

[2] K. Corrádi and I. Kátai, A note on K. S. Gangadharan's paper "Two classical lattice point problems", Magyar Tud. Akad. Mat. Fiz. Tud. Oszt. Kötzl. 17 (1967), 89-97 (in Hungarian).

[3] K. S. Gangadharan, Two classical lattice point problems, Proc. Cambridge Philos. Soc. 57 (1961), 699-721.

[4] S. W. Graham and G. Kolesnik, Van der Corput's Method of Exponential Sums, Cambridge Univ. Press, Cambridge, 1991.

[5] J. L. Hafner, New omega theorems for two classical lattice point problems, Invent. Math. 63 (1981), 181-186.

[6] G. H. Hardy, On the expression of a number as the sum of two squares, Quart. J. Math. 46 (1915), 263-283.

[7] M. N. Huxley, Exponential sums and lattice points II, Proc. London Math. Soc. 66 (1993), 279-301.

[8] —, Area, Lattice Points, and Exponential Sums, London Math. Soc. Monographs (N.S.) 13, Oxford, 1996.

[9] I. Kátai, The number of lattice points in a circle, Ann. Univ. Sci. Budapest. Eötvös Sect. Math. 8 (1965), 39-60.

[10] E. Krätzel, Lattice Points, Deutsch. Verlag Wiss., Berlin, 1988.

[11] -, Bemerkungen zu einem Gitterpunktproblem, Math. Ann. 179 (1969), 90-96.

[12] G. Kuba, On sums of two $k$-th powers of numbers in residue classes II, Abh. Math. Sem. Univ. Hamburg 63 (1993), 87-95.

[13] M. Kühleitner, W. G. Nowak, J. Schoissengeier and T. Wooley, On sums of two cubes: an $\Omega_{+}$-estimate for the error term, Acta Arith. 85 (1998), 179-195.

[14] W. G. Nowak, On sums of two $k$-th powers: a mean-square bound for the error term, Analysis 16 (1996), 297-304.

[15] - Sums of two $k$-th powers: an Omega estimate for the error term, Arch. Math. (Basel) 68 (1997), 27-35.

[16] D. Redmond, Mean value theorems for a class of Dirichlet series, Pacific J. Math. 78 (1978), 191-231.

[17] L. Schnabel, Über eine Verallgemeinerung des Kreisproblems, Wiss. Z. FriedrichSchiller-Univ. Jena Math.-Natur. Reihe 31 (1982), 667-781.

[18] J. D. Vaaler, Some extremal problems in Fourier analysis, Bull. Amer. Math. Soc. 12 (1985), 183-216.

[19] J. G. van der Corput, Over roosterpunkten in het plate vlak, thesis, Groningen, 1919 .

Institut für Mathematik

Universität für Bodenkultur

A-1180 Wien, Austria

E-mail: kleitner@edv1.boku.ac.at 